\title{
Basics of Technique Evaluation the Use of Hydroelectric Resources in Their Work in EPS
}

\author{
Anastasia G. Rusina*, Tamara A. Filippova, \\ Ekaterina A. Savban and Jahongir K. Khujasaidov \\ Novosibirsk State Technical University \\ 20 Karl Marks, Novosibirsk, 630073, Russia
}

Received 28.04.2016, received in revised form 31.08.2016, accepted 28.12.2016

\begin{abstract}
Hydroelectric power stations exert a great influence on distribution of the electric power on regional and, especially in the wholesale electrical power markets in models of the type "water resources - electricity consumption". In this article outlines some principles of creation a technique of calculations for hydroelectric power stations. We will call model of such type of $R-P-L$ (resources, production, load) of hydroelectric power station. Considering uncertainty of a drain of hydroelectric power station and information difficulties, tasks traditionally is divided into two parts, depending on the planning period - the long-term and short-term modes. In the scheme of use of the R-P-L model there are a lot of features and changes in comparison with the last developments executed for Unified Energy System.
\end{abstract}

Keywords: hydropower resources, development, optimization, efficiency, energy balance, forecasting.

Citation: Rusina A.G., Filippova T.A., Savban E.A., Khujasaidov Ja.K. Basics of technique evaluation the use of hydroelectric resources in their work in eps, J. Sib. Fed. Univ. Eng. technol., 2017, 10(3), 426-434. DOI: 10.17516/1999-494X-2017-10-3-426434.

(C) Siberian Federal University. All rights reserved

* Corresponding author E-mail address: rusina@corp.nstu.ru, ta filippova@ngs.ru 


\title{
Основы методики оценки
}

\author{
использования ресурсов ГЭС \\ при функционировании \\ электроэнергетической системы
}

\author{
А.Г. Русина, Т.А. Филиппова, \\ Е.А. Совбан, Д.Х. Худжасаидов \\ Новосибирский государственный технический университет \\ Россия, 630073, Новосибирск, пр. Карла Маркса, 20
}

\begin{abstract}
Гидроэлектростанции (ГЭС) оказывают большое влияние на распределение электроэнергии на региональных и особенно на оптовых электроэнергетических рынках в моделях вида гидроресурсы - потребление электроэнергии. В данной статье излагаются некоторые принципы создания модели вида ресурсы-производство-нагрузка (Р-П-Н) для обоснования методикиучётарежимов работы ГЭС в электроэнергетических системах (ЭЭС). Учитывая неопределенность стока ГЭС и информационные сложности, задачи традиционно делятся на две части в зависимости от периода планирования - долгосрочные и краткосрочные режимы. В схеме использованиямодели Р-П-Нмного особенностей и изменений по сравнению с прошлыми разработками, выполненными для единой энергетической системы.
\end{abstract}

Ключевые слова: гидроэнергетические ресурсы, выработка, оптимизация, эффективность, энергетический баланс, прогнозирование.

\section{Введение}

Задача управления режимами ГЭС очень сложна. Цель ее решения - рациональное использование водных ресурсов. Гидроэнергетические ресурсы ГЭС изменяются в широких пределах, и их рациональное использование зависит от величины стока, гидрографа реки, требования энергосистемы к ГЭС, технологического процесса режима оборудования и многих других факторов.

Оптимальное управление режимами ГЭС осуществляется в условиях неполноты и недостаточной достоверности исходной информации. Имеются элементы случайности в формировании естественного режима стока, графики нагрузки системы, в значениях располагаемых мощностей, в составе работающего оборудования электростанций и используемых энергетических ресурсов.

Прогнозирование исходной информации на сравнительно короткие промежутки времени (до месяца) в настоящее время особых затруднений не вызывает. При этом не исключается, что отдельные факторы, например графики нагрузки системы, под влиянием случайных обстоятельств отклоняются от прогнозируемых.

При расчете длительных режимов регулирования стока гидрологическая информация носит вероятностный характер и никакое другое предсказание стока на сезон и год (многолетний период) не может быть правомерным [1].

Современные гидростанции обычно работают в составе водохозяйственного комплекса (ВХК). Роль ГЭС в различных ВХК неодинакова. В некоторых комплексах ведущая роль при-

$$
-427-
$$


надлежит энергетике, в других - основным участником является иная отрасль. В таких случаях ставится задача рационального распределения водных ресурсов. Режим ГЭС будет находиться в прямой связи с теми требованиями неэнергетических участников комплекса, которые ей предписано выполнять. Управление режимами ГЭС в условиях жестких водохозяйственных ограничений приводит к дополнительным затруднениям. В водохозяйственных системах по мере их развития требования обычно возрастают и энергетическая значимость гидроузла уменьшается.

На текущий момент регулировочный диапазон станций ограничен водохозяйственными требованиями. В связи с этим появляется необходимость анализа и изменения методов и задач оптимального использования ресурсов ГЭС.

\section{Особенности алгоритмизации модели Р-П-Н.}

На основе анализа функционирования ГЭС в составе ЭЭС была предложена алгоритмическая структура модели Р-П-Н.

Блок 1 - информационное пространство. Принципиальным вопросом является необходимость использования гидроэнергетической и гидравлической информации по напорам, стоку, расходам, уровням бьефов, что увеличивает не только размер массивов традиционной информации тепловых электроэнергетических систем, но и неопределенность всех задач планирования режимов гидротепловых ЭЭС. В блок включаются модели прогнозирования, так как без прогнозов невозможно решать режимные задачи ЭЭС.

Блок 2 - гидроэнергетический режим, в котором определяется оптимальное использование водных ресурсов ГЭС (бытовой приточности и запасов стока в водохранилище).

Главная задача - как использовать гидроэнергетические ресурсы и получить максимум выработки электроэнергии. Это означает, что требуется получить максимум КПД по напору и по расходу. Оптимизация производится по критерию максимума выработки электроэнергии за полный цикл гидроэнергетических расчетов, т.е. за гидрологический год. Это отвечает условию поздней сработки водохранилища в период межени и раннему заполнению водохранилища в период паводка. Если рассматривается каскад ГЭС, то должен быть добавлен критерий максимума напора нижележащей станции каскада. Все вышесказанное соответствует рациональному ведению бизнеса и максимуму продаж электроэнергии ГЭС и оптимизации режима ЭЭС.

В блоке решается комплекс задач по рациональному использованию гидроэнергетических ресурсов одиночных и связанных станций в каскаде. Выработка электроэнергии на ГЭС зависит от стока, а режим использования стока в свою очередь существенно зависит от напора. Главную роль играет критерий оптимизации выработки электроэнепргии. Критерий

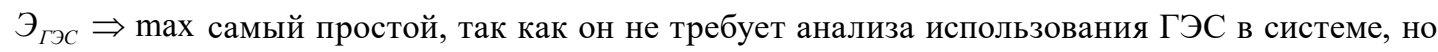
решение является заведомо не наилучшим.

Блок 3 - технологический процесс ГЭС. В нем рассматриваются задачи внутристанционных решения выбора состава и режима гидроагрегатов при заданной нагрузке станции по критерию максимума КПД. Это основной показатель технологического процесса преобразования

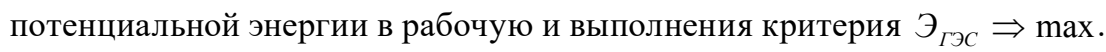

КПД ГЭС является произведением КПД по напору $(\mathrm{H})$, расходу $(\mathrm{Q})$ и технологическому процессу: 


$$
\begin{aligned}
& \eta_{\Gamma \ni C}=\eta_{H} \eta_{Q} \eta_{\text {mex }}, \\
& N_{\text {подв }}=k Q H \text {, } \\
& \eta_{H}=\frac{H_{\text {полезн }}}{H_{\text {подв }}}=\frac{H_{\text {подв }}-\Delta H}{H_{\text {подв }}}, \\
& \eta_{Q}=\frac{Q_{\text {полезн }}}{Q_{\text {подв }}}=\frac{Q_{\text {подв }}-\Delta Q}{Q_{\text {подв }}}, \\
& \eta_{\text {mехн }}=\frac{N_{\text {полезн }}}{N_{\text {подв }}}=\frac{N_{\text {подв }}-\Delta N}{N_{\text {подв }}},
\end{aligned}
$$

где $\Delta H, \Delta Q$, и $\Delta N-$ потери соответственно напора, расхода и мощности ГЭС или изменчивость этих факторов, влияющих на КПД.

Полный КПД турбинной установки по напору:

$$
\eta_{H}=\frac{H_{\text {полезн }}}{H_{\text {полезн }}-\Delta H_{\text {нб}}-\Delta H_{\text {в.c }}-\Delta H_{\text {турб }}-\Delta H_{\text {o.c }}}=\eta_{H_{\text {нб}}} \cdot \eta_{H_{\text {в.c }}} \cdot \eta_{H_{\text {турб}}} \cdot \eta_{H_{\text {o.c }}}
$$

Здесь индексы: нб - нижний бьеф, вс - водоподводящие сооружения, турб - турбина, ос отводящие сооружения.

Блок 4 - режим ГЭС в ЭЭС и блок 5 - коммерческий баланс.

В ЭЭС должно соблюдаться условие получения минимума издержек с учетом структуры мощностей ЭЭС. Основные особенности ЭЭС соответствуют ее энергетическим балансам, и критерием оптимизации является минимизация суммарных издержек всех единиц (станций и сетей). Такая оптимизация производится для календарного года при изменении нагрузки.

Во многих работах показано, что режим с критерием максимума выработки энергии не всегда соответствует режиму с критерием минимума издержек, причем результат в первом случае хуже на несколько процентов. Ввиду этого всегда отдавалось предпочтение критерию минимума издержек. Однако сейчас это связано с большими информационными трудностями и есть две альтернативы: либо режим рассчитывается для станции и тогда необходима системная информация, либо режим рассчитывается для системы и тогда необходима гидрологическая информация.

Целесообразно этот расчет выполнять по схеме последовательного уточнения режима. Сначала для ГЭС рассчитывается режим по критерию максимума выработки энергии. Вторым этапом является его дооптимизация по критерию минимума издержек и, наконец, есть третий этап по максимуму прибыли продажи на электроэнергетическом рынке. Конечно, при этом будут определенные потери и эффективность использования потенциальных ресурсов понизится, но при таком подходе снимается часть информационных проблем.

Топливная эффективность ресурсов при использовании ГЭС в ЭЭС-фактор, имеющий важнейшее значение для режимов ЭЭС.

Понятие «топливная эффективность» отражает влияние ГЭС на энергетические балансы ЭЭС, поскольку очевидно, что ГЭС дает всегда определенную выгоду для системы - и по режиму тепловых станций особенную $[2,3]$. В балансах мощности гидростанции размещаются чаще всего без учета эффективности энергоресурсов. 
Топливная эффективность гидроресурсов (расхода воды и стока) зависит в основном от показателей энергетического баланса мощностей ЭЭС. Баланс мощностей изменяется ежедневно, и по времени года, соответственно, изменяется и топливная эффективность гидроресурса. Для определения топливной эффективности используется характеристика относительных приростов ЭЭС, данные баланса мощностей и места станции в балансе (пик, полупик, база). При этом изменяются относительные приросты по топливу и издержкам.

$$
\frac{\Delta B}{\Delta P}, \frac{\Delta H}{\Delta P} . F=\frac{C_{1}}{T_{K M N}} .
$$

Топливная эффективность позволяет изменить содержание и структуру себестоимости электроэнергии ГЭС:



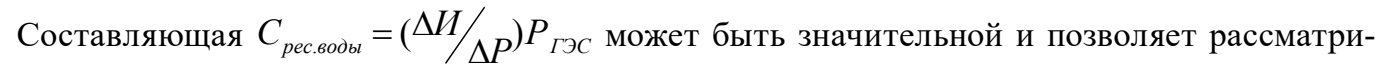
вать многие режимные задачи ЭЭС с учетом стоимости гидроресурса.

Необходимо учитывать и эффективность распределения нагрузки между станциями ЭЭС. Такая задача детально разработана.

Полный алгоритм включает 5 основных блоков, перечисленных выше: информационный, гидроэнергетический, технологический, энергетических балансов гидротепловой ЭЭС, коммерческий. Расчет режима ведется от базового первоначального плана к конечному, при этом режим ГЭС рассчитывается последовательно по трем критериям: максимум выработки энергии, минимум издержек, максимум прибыли.

\section{Показатели оценки режимов ГЭС в модели Р-П-Н}

В настоящее время при учете бизнес-интересов главными показателями являются прибыль и цена. Ошибочность этого раскрыта во многих работах, например в [4].

Показатели эффективности гидростанций могут рассчитываться с различными целями, и от этого зависит их вид и содержание. Все без исключения показатели эффективности работы ГЭС зависят от ее выработки электроэнергии, и требуется решение многих задач.

Структурная модель производства электроэнергии на ГЭС должна учитывать весь процесс от ресурсов производства до реализации электроэнергии по всем видам деятельности.

Использование ресурсов электроэнергии. Гидроэнергетический потенциал зависит от параметров станции, стока реки, режима использования водных ресурсов. Выработка электроэнергии определяется расчетами по данным водных ресурсов, по режимам напора, по КПД технологического процесса станции. Все составляющие переменны во времени и имеют высокий уровень неопределенности.

Транспорт электроэнергии и режим передачи электроэнергии сетевыми компаниями СК могут иметь разные масштабы и хозяйственные формы $[5,6]$. Сетевые компании и предпри-

$$
-430-
$$


ятия, осуществляющие транспорт электрической энергии, - это перепродавцы, и цена всех і предприятий будет $L_{\text {mранс }}=\sum L_{m p i}$. Обычно ГЭС не имеет свободы выбора трасс транспорта и не располагает данными по их определению. Тогда требуется разработка методики создания электрической схемы или специальной методики прогнозирования для оценки затрат на транспорт. Часть транспортных задач ГЭС может решать самостоятельно.

\section{Методика расчета показателей и оценки деятельности ГЭС}

Рамки статьи не позволяют подробно остановиться на методике расчета показателей эффективности, но очевидно, что без специального алгоритма она вряд ли может быть реализована. Дадим отдельные фрагменты такой методики.

Рассмотрим содержательно понятие КПД по напору, что требует детализации понятий «подведенный напор» и «полезный напор»:

$$
\eta_{H}=\frac{H_{\text {полезн }}}{H} .
$$

Напор блока для плотинных ГЭС

$$
H_{б л}=Z_{B Б}-Z_{H Б} \text {. }
$$

Для деривационных ГЭС напор

$$
H_{\text {б̆ }}=Z_{B Б}-Z_{H Б}-\Delta H_{\text {дер }} \text {. }
$$

Напор, подведенный к турбине, меньше напора блока на величину потерь в водоподводящих сооружениях, которые можно заранее рассчитать:

$$
H_{\text {подв. мурб. }}=H_{\text {бл }}-\Delta H_{B C} \text {. }
$$

Полезный напор турбинной установки меньше напора блока на величину потерь напора в турбине и в отводящих сооружениях:

$$
H_{\text {полезн }}=H_{\text {подв. пурб. }}-\Delta H_{\text {турб. }}-\Delta H_{\text {отв }} .
$$

Полный КПД турбинной установки по напору

$$
\begin{aligned}
& \eta_{H}=\frac{H_{\text {полезн }}}{H}, \\
& \eta_{H}=\frac{H_{\text {полезн }}}{H_{\text {полезн }}+\Delta H_{\text {бл }}+\Delta H_{\text {в.с }}+\Delta H_{\text {турб }}+\Delta H_{\text {o.c }}}=\eta_{\text {H.б.л }} \eta_{\text {H.в.с }} \eta_{\text {H.турб }} \eta_{\text {H.о.с }} .
\end{aligned}
$$

Со временем напорные характеристики изменяются.

КПД по расходу может составляться по верхнему бьефу, а для каскада - и по нижнему.

Уравнение баланса имеет следующий вид:

$$
Q_{в \tilde{\sigma}}(t)=Q_{n p}(t) \pm Q_{6}(t)+Q_{o c}(t)-Q_{u c n}(t) \pm Q_{n}(t)-Q_{\phi}(t)-Q_{в x}(t),
$$

где $Q_{n p}$ - приточность; $Q_{s}$ - запасы водохранилища расход; $Q_{o c}-$ осадки; $Q_{u c n}-$ испарения с его поверхности; $Q_{n}$ - потери на ледообразование; $Q_{\phi}-$ потери на фильтрацию через ложе водохранилища; $Q_{6 x}-$ забор воды на нужды водопотребителей и водопользователей. 
Баланс расходов в нижнем бьефе станции

$$
Q_{\mu \tilde{\sigma}}(t)=Q_{\Gamma \ni C}(t)+Q_{x . c \tilde{\sigma}}(t)+Q_{u л}(t)+Q_{\phi}(t)+Q_{n p}(t),
$$

где $Q_{\Gamma \ni с}$ - расход воды через турбины ГЭС; $Q_{x . c \sigma}-$ расход холостых сбросов через водосбросные сооружения; $Q_{u л}$ - расход на шлюзование; $Q_{\phi}$ - фильтрация воды через гидротехнические сооружения и различные устройства станции; $Q_{n p}-$ расход воды, поступающий в нижний бьеф через рыбоходы, ледосбросы и другие сооружения.

Баланс расходов по станщиии

$$
Q_{\beta \sigma}(t)=Q_{\mu \sigma}(t)
$$

Часто составляющие $Q_{o c}, Q_{u c n}, Q_{n}, Q_{\phi}, Q_{6 x}$ учитывают в виде поправок к бытовой приточности, и тогда уравнение баланса записывается в общепринятом виде:

$$
Q_{\mu \sigma}(t)=Q_{\Gamma \ni C}(t)+Q_{x . c \sigma}(t) .
$$

Баланс расходов для каскада ГЭС

Для каскада из n станций уравнения баланса представляются системой уравнений:

$$
Q_{\mu \bar{\sigma} . n}(t)=Q_{\mu \tilde{\sigma} . n-1}\left(t-\tau_{n-1}\right) \pm Q_{k n}(t)+Q_{\sigma . n p . n}(t) .
$$

Из приведенных уравнений легко определить факторы, влияющие на КПД по расходу.

КПД технологического проиесса по мощности учитывает преобразование подведенной энергии в рабочих органах турбины генератора и водоподводящих сооружениях.

Подведена мощность $\mathrm{N}_{\text {подв}}$, мощность на зажимах генератора будет $\mathrm{N}_{\text {пол }}$, КПД агрегатного блока

$$
\eta_{a}=\eta_{T} \eta_{\Gamma} \eta_{B C},
$$

где индексы т - турбина, г - генератор, а $\eta_{B C}-$ КПД водоподводящих сооружений.

В заключение необходимо подчеркнуть, что требуется специальная методика определения всех составляющих КПД и факторов, причем не стартовых условий, а фактических. Это сложная информационная задача.

\section{Себестоимость электроэнергии ГЭС}

Удельные издержки производства на действующей ГЭС

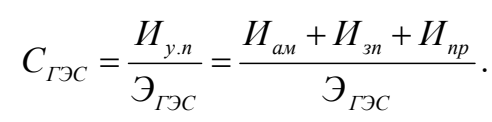

Экономическая эффективность ГЭС - это минимум себестоимости. Ее величина зависит от выработки электроэнергии.

Самое главное, что цена продажи электроэнергии определяется в прямой зависимости от себестоимости. 
Коммерческая эффективность электроэнергии

Оптовый рынок электроэнергии функционирует по регламентам, выработанным организаторами рынка. Они не координируются с технологическим процессом преобразования гидроэнергии на различных этапах. Вместе с тем нельзя исключить этот этап в комплексной оценке эффективности использования ресурсов ГЭС.

Первоочередным является показатель максимальной выработки электроэнергии ГЭС, что достигается за счет оптимизации: напора, расхода, КПД техники и технологии, топливной эффективности, распределения нагрузки в ЭЭС, потерь электроэнергии в сетях, коммерческих преимуществах в отношениях купли/продажи. А в электроэнергетической системе от выработки энергии на ГЭС зависят функции остальных станций, контроль и анализ затрат управления системой.

Эффективность использования гидростанщий в комплексе

Эффективность включает два компонента - затраты и выгоду. Затраты - это себестоимость $C_{\text {гэс }}$, выгода - режимная эффективность, которую дает ГЭС при работе в ЭЭС, и коммерческая эффективность, которую получает ГЭС как субъект электроэнергетического рынка.

Тогда суммарная эффективность:

$$
S=k_{c} C-\left(\sum_{j} k_{j} \Delta \ni_{э \phi . j}\right)
$$

Показатели эффективности могут иметь различные единицы измерения КПД в процентах, энергия в кВтч, себестоимость в рублях, и их надо приводить к единой форме с использованием множителей приведения $\mathrm{k}$.

Практическое значение показателей эффективности. Наиболее возможными являются следующие практические области применения показателей эффективности:

- Комплексная эффективность позволяет сравнивать станции между собой, сравнивать их с нормативами в целом.

- Сравнение возможно и по отдельным этапам использования гидроресурсов, и по отдельным задачам.

- Проведение анализа и планирование путей энергосбережения.

\section{Выводы}

Определение оптимального режима ГЭС и увязка его с существующими требованиями энергосистемы - необходимые условия составления актуальной схемы использования водных ресурсов ГЭС. Одним из основных технико-экономических показателей ГЭС в период эксплуатации выступает себестоимость выработанной электроэнергии. В некоторых случаях требования системы к ГЭС могут значительно превосходить все ее потенциальные возможности, но и тогда может быть получен существенный эффект в результате правильного критерия и схемы регулирования. Эффект оптимизации режимов ГЭС определяется не только характеристиками оборудования, но и водными режимами гидростанций. На режим ГЭС накладываются ограничения водохозяйственного комплекса, что изменяет ее функции

$$
-433-
$$


и режим рабочих мощностей, что в свою очередь отражается на роли ГЭС на электроэнергетическом рынке.

Приведенная методика по теме эффективность использования ГЭС от ресурсов до коммерческих результатов является актуальной и для действующих станций, и для проектируемых. Сейчас предполагается проектирование и создания новых ГЭС, и эта методика влияет на инвестиционный проект.

Оценка эффективности использования гидроресурсов имеет большую актуальность и для цен на электроэнергетическом рынке. Приведем пример по ОЭС Сибири, в балансах которой участвует Новосибирская, Красноярская, Братская, Усть-Илимская, Саяно-Шушенская и Иркутская гидростанции. У них разные напоры - примерно от 20 до 200 м. И единица объема стока на Новосибирской ГЭС имеет примерно в 10 раз меньший потенциал энергии, чем на Красноярской и в 20 раз меньший, чем на Саяно-Шушенской, при небольшой различия в себестоимости энергии. А при своих ценовых заявках станции исходят только из себестоимости. Потенциал практически не учитывается на станции, и даже в ОЭС Сибири он неизвестен.

Для эксплуатируемых ГЭС важно правильно оценивать их результативность не только по заявленным ценам, а с учетом их связи цен с тем, как они используют ресурсы, возобновляемые и дешевые, гидроресурсы - это национальное богатство. Тема, рассмотренная в работе, требует ее глубокого исследования.

\section{Список литературы}

[1] Филиппова Т.А., Мисриханов М.Ш., Сидоркин Ю.М., Русина А.Г. Гидроэнергетика: учеб. пособие. Изд. 3-е, перераб. Н.: Изд. НГТУ, 2013, 620 с. [Filippova T.A., Misrikhanov M.Sh., Sidorkin Iu.M., Rusina A.G. Hydropower: a tutorial. Edition 3, revised. Novosibirsk, Izd. NGTU, 2013, 620 p. (in Russian)]

[2] Sekretarev Y., Sultonov Sh., Shalnev V. Optimal Control Mode of the Vakhsh Hydropower Reservoirs to Reduce Electricity Shortages in Tajikistan, Applied Mechanics and Materials, 2015, 792, 446-450.

[3] Allen J., Bruce F., Gerald B. Power Generation Operation and Control. Third ed. New York, John Wiley \& Sons, 2013. 656 p.

[4] Русина А.Г. Развитие теории и методологии анализа электроэнергетических систем для управления установившимися режимами. Докторская диссертация. Томск, 2013. 297 c. [Rusina A.G. Development of the theory and methodology of analysis electric power systems for management established modes. Doctoral thesis, Tomsk, 2013. 297 p. (in Russian)]

[5] Русина А.Г., Филиппова Т.А. Режимы электрических станций и электроэнергетических систем. Н.: Изд. НГТУ, 2014. 399 с. [Rusina A.G., Filippova T.A. The modes of power plants and electric power systems. Novosibirsk, Izd. NGTU, 2014. 399 p. (in Russian)]

[6] Легалов Д.И. Методика планирования долгосрочных оптимальных режимов электроэнергетических систем с ГЭС, основанный на методе динамического программирования. Кандидатская диссертация. Иркутск, 2005. 139 с. [Legalov D.I. Methods of long-term planning of optimal modes of power systems with HPPs, based on the method of dynamic programming. $\mathrm{PhD}$ thesis, Irkutsk, 2005. 139 p. (in Russian)] 